\title{
EFFETS D'UNE PÉDAGOGIE DES AUTOROUTES DE L'INFORMATION DANS LE DOMAINE DES COLLECTIVITÉS TERRITORIALES
}

\author{
Catherine Loneux ${ }^{1}$
}

Nous proposons de réfléchir à la relation entre les savoirs informels et les médias, à travers l'exemple précis d'analyse de corpus, "Autoroutes de l'information et territoires", bimensuel qui représente depuis sa création en 1996 un vecteur d'information et de savoir unique, plus ou moins élaboré, destiné surtout aux élus et personnels des collectivités territoriales spécialisés dans le domaine des nouvelles technologies. Il s'adresse aussi à des professionnels touchant de près ce secteur mais qui l'utilisent davantage comme un moyen d'observation, de veille informationnelle sur les collectivités locales françaises, n'ayant pas un usage pédagogique de la publication. Elle est exemplaire dans ce domaine, et il n'existe pas d'équivalent pour les entreprises privées.

Les évolutions technologiques, réglementaires, les mutations technologiques majeures dans notre société encouragent le développement des technologies de communication dans le secteur des collectivités locales. Le rapport entre ces médias et le savoir a déjà été

1 CERCOR, Université de Rennes 2.

Recherches en communication, $\mathrm{n}^{\circ} 15$ (2001). 
mis en évidence concernant certaines sphères de la société. Nous nous concentrerons ici sur le secteur des collectivités territoriales, qui n'échappe pas à ce phénomène : le récepteur reçoit le média comme une connaissance, une dynamique s'impose, entre le savoir et la communication.

Un savoir qui n'a pas pu être enseigné dans le cadre de l'Université ou d'autres formations, peut s'imposer par une voie non formelle à une sphère donnée, tel est l'objectif de notre première partie.

Notre seconde partie s'attachera à comprendre les effets de ces connaissances sur la sphère des professionnels des collectivités locales : comment peuvent-elles avoir une incidence significative en termes de structuration?

Sur le plan méthodologique, nous nous inscrivons dans la lignée des études sur l'influence des technologies de l'information et de la communication sur les groupes sociaux, rejoignant la critique anthropologique sur les technologies, ou la critique plus sociale sur leurs effets. Nous avons choisi arbitrairement d'examiner les numéros de l'année 1999, et procéderons à une analyse de ces données écrites en isolant des thèmes, les ordonnant selon un ordre construit pour notre propos. De manière systématique, percent derrière les écrits les intentions pédagogiques des auteurs. En effet, les savoirs informels finissent par avoir un effet unificateur et institutionnalisant au sein de la population touchée. Cadres territoriaux, élus, techniciens, etc., chacun de ces groupes tire parti, à sa façon et à des degrés plus ou moins élevés de cette pédagogie proposée par "Autoroutes de l'information et territoires". Des entretiens conduits avec les responsables éditoriaux de ce support ou des élus enrichiront ce travail.

Sur un plan conceptuel, en dehors de la notion de savoir informel que nous proposons de relier à ce support, en examinant ses modes de diffusion, d'autres concepts pourront aussi être abordés, lorsque nous traiterons des effets structurants de ces savoirs dans les collectivités territoriales : la création d'un monde social, ou l'expertise professionnelle du point de vue d'une observation anthroposociologique. 


\section{Savoir informel et média : un lien revendiqué du côté des concepteurs}

La forme du savoir est rendue possible par le média lui-même, qui offre aux élus et personnels spécialisés (informaticiens, responsables des Systèmes d'Information Géographiques, Observatoire des Télécoms dans la Ville, etc.), des informations sur l'internet et ses possibles utilisations.

Il existe d'autres manières d'apprendre pour les élus et les personnels des collectivités locales, et la presse s'impose comme un vecteur important de d'information et de formation continue pour eux. Les thèmes, contenus, et paysages conceptuels répondent à une volonté de vulgarisation des savoirs sur les nouvelles technologies, à l'intérieur de rubriques dont la vocation pédagogique et la fonction de partage des connaissances sont affirmées.

Plusieurs questions nous animent: favorable)

1. Pourquoi ce nouveau vecteur de formation? (un contexte

2. Quelle forme prend-t-il ? (un support pédagogique)

3. En quoi ce savoir est-il informel ?

\section{Un contexte favorable}

L'administration et la manière de travailler dans les Collectivités Territoriales ont changé, orientées vers une dématérialisation des procédures, qui comporte des enjeux démocratiques autant du côté des citoyens et de leur place dans la société que du côté des élus, qui doivent s'adapter et comprendre ces évolutions ${ }^{1}$.

Depuis les lois de décentralisation françaises de 1982 et 1983, les pouvoirs publics ont infléchi leur mode d'action, et les politiques locales s'orientent vers l'animation économique plutôt que l'action défensive. Cette attitude révèle une mutation de la perception du champ de l'action publique. L'espace de l'action publique n'en est

1 J.-P. BASQUIAT, Internet et les administrations - la grande mutation, Paris, BergerLevrault, 1999, p. 33. 
pas pour autant réduit, mais il change de nature. L'animation des réseaux de jeux d'acteurs, devient la préoccupation centrale des élus en matière d'intervention économique publique.

Le modèle territorial s'inspire du modèle fonctionnel de l'entreprise et va être victime d'une mutation progressive. Les valeurs nouvelles autour du libéralisme économique se déclinent en terme de marché, d'entreprise, de rentabilité, de profit, et surtout de nouvelles technologies.

\section{Un support pédagogique peut aider à la compréhension de ce changement.}

Les concepteurs de la revue ont senti ce mélange de curiosité et de réticence vis-à-vis des technologies de communication, et ont su imaginer une formule attrayante pour un certain lectorat. Voir les structures qui organisent l'accès aux savoirs, c'est voir les transformations de la forme des savoirs.

De nouvelles formes d'acquisition des savoirs ont émergé à l'attention des différents acteurs, souvent peu formés -voire même réticents- à l'utilisation des technologies, et un tel support a pu aisément devenir instrument pédagogique et didactique.

"Autoroutes de l'information et territoires" médiatise les recompositions organisationnelles et représente un mode de réflexion et $d$ 'appropriation original, que les concepteurs revendiquent comme pédagogique.

Les thèmes, contenus, et paysage conceptuel offerts répondent à une volonté de vulgarisation des savoirs sur les nouvelles technologies, à l'intérieur de rubriques dont la vocation pédagogique et la fonction de partage des connaissances sont affirmées. Le "salon des maires de France" par exemple, sert à renforcer ce type de supports de presse, en proposant aux maires une utilisation possible des technologies, une relation à l'innovation, une dynamique qui articule le stade de l'apprentissage puis celui de la communication.

La ligne éditoriale est clairement orientée vers la divulgation du savoir. Une lettre d'information professionnelle existe dans le privé, avec "Le quotidien multimédia", qui suit l'actualité en matière de technologie, mais elle n'a pas cette vocation pédagogique. 


\section{Thèmes et rubriques}

Une analyse par thèmes et par rubriques relèvera le vocabulaire didactique et pédagogique utilisé, les orientations du discours, les récurrences sémantiques, etc.

L'ordonnancement des rubriques, d'abord, est quasi immuable : "A savoir", "Régions", "Expériences", "Dossier", "Partenaires", "Bonnes adresses". Toutes n'ont pas vocation à être pédagogique. Certaines sous-rubriques sont purement factuelles, comme "Boîte à idées", "En bref", ou bien publicitaires, comme "Partenaires", qui vante les avantages de tel logiciel, de telle entreprise, et présente les services, produits, et sociétés, qui peuvent aider les collectivités dans la mise en place de projets technologiques. Seules les rubriques qui sont attachées à développer du savoir retiendront bien sûr notre attention.

"A savoir", par exemple, est une rubrique d'explication des termes techniques, de vulgarisation scientifique et technique, qui sert à donner des ressources pour construire des projets, des statistiques, des explications sur les mots clefs. "Bonnes adresses" répond à la même préoccupation. La nouvelle rubrique, "Régions", propose des exemples d'implantation des technologies, avec des "recettes" de ces réussites, sur les régions et leurs politiques de développement en la matière. Elle a fait paraître déjà des enquêtes sur le LanguedocRoussillon, le Nord-Pas-de-Calais, les Pays de Loire, l'Alsace.

Toujours dans ce souci d'encouragement des initiatives dans les choix de technologie, la rubrique "Expérience" montre aux collectivités les pratiques des collectivités voisines : aspects organisationnels, techniques, objectifs, coûts, aspects pratiques, adresses personnelles, des coordonnées utiles, etc. Une collectivité de taille similaire pourrait avoir envie de se nourrir d'une telle expérience et de s'y identifier. L'idée de reproductibilité des modèles est contenue. "Dossier" est la rubrique qui suit, attachée à traiter un cas ou un problème particulier.

Luc Derriano, rédacteur en chef, souhaite vulgariser les savoirs sur les technologies, à l'intérieur de rubriques dont la "vocation pédagogique et la fonction de partage des connaissances sont affirmées"1. Il propose pour cela une hiérarchie spécifique dans le discours.

Il veut d'abord expliquer, pour contribuer à l'instruction des élus, leur faciliter le travail, les repérer dans le maquis des législations sur

1 L. Derriano, entretien du 17 nov. 2000. 
les nouvelles technologies dans les villes. Tout est présenté dans des schémas extrêmement ramassés, clairs, simples, dépeignant par exemple, pour une affaire donnée, les acteurs, leur rôle, leur action, etc. Les informations financières ont une place de choix : le coût des opérations, des installations, afin d'être reçus par les élus.

Il veut aussi encourager certains usages de manière un peu plus enthousiaste, afin d'orienter les choix en matière de technologies pour un changement possible, et d' "aider à l'appropriation des outils" (AIT, $n^{\circ} 42,15 / 01 / 99$ ). Privilégier le net, transférer, initier aux tendances dans le développement des nouvelles technologies. Faire connaître les moyens, les modes de financement possible pour accéder aux nouvelles technologies ("bornes d'accès internet dans les missions locales" (AIT, n 42, 15/01/99). Il précise à quoi servent les projets, et donne les recettes aux communes pour éviter certaines erreurs dans la mise en place des technologies au service des usagers ("Quelle formation pour les animateurs ?", "Les dérives des utilisateurs", etc.). Des cas pratiques exposés décrivent les phases d'implantation des projets : "Méthode - Matériel", "un point sur les offres de providers", "conseils pour réussir" (AIT, n 49, 17/05/00). Tous les moyens sont offerts pour renforcer les savoir-faire acquis : conseils en ingénierie de l'information, sur les logiciels, les webs, les formations possibles, etc.

De nouvelles compétences apparaissent, et requièrent des qualifications qu'il faut souvent chercher à l'extérieur des collectivités locales et pour lesquelles la formation est indispensable, et peut être prise en charge, entre autres, par ce support papier.

Les relations de réseaux se forment aujourd'hui à partir d'une proximité professionnelle plutôt que physique. Les hommes de même métier ont à se retrouver et à nouer des relations inter-personnelles audelà des frontières. Ainsi, le traitement de l'information, la mise en commun des ressources cognitives sur un métier, font partie des axes d'actions possibles pour les collectivités territoriales ${ }^{1}$. Des relations de réseaux spontanées émergent par le biais d'institutions et régulent l'échange d'information entre acteurs par une politique d'animation visant à pallier les carences dans la circulation de l'information, à aider à procéder à des sauts technologiques, à accompagner les ruptures dans les savoir-faire traditionnels.

1 P. Musso (dir.), Communiquer demain, Paris, Éd. Datar, 1997, p. 10. 
Ces savoirs sont simples, résumés, proche d'une presse de vulgarisation, mais les modalités de représentation de l'Internet qu'ils proposent posent question : socialement, nous sommes censés être face à une source d'information, qui devient source de savoir. Comment cela opère-t-il ?

\section{En quoi ce savoir est-il informel ?}

Identifier le savoir formel du savoir informel consiste à examiner les conditions sociales de communication et de réception. A voir l'accessibilité, l'énonciation, la mise en forme sémiotique, le type de médiatisation.

\section{Savoir ludique}

Ce savoir est informel d'abord parce que le dispositif de communication des savoirs proposé transforme la forme d'acquisition des savoirs en donnant une vision orientée de cet enseignement, qui ne doit pas avoir l'air d'être un enseignement et en offrant une dimension nouvelle du savoir, tournée vers le ludique, évitant le caractère austère des vecteurs traditionnels de savoir.

\section{Savoir non identifié}

Ce savoir est informel aussi parce qu'il se cache. La traduction, au sens sociologique, est une opération particulière qui transforme un énoncé problématique particulier dans le langage d'un autre énoncé particulier. Le savoir informel diffusé par les experts rédacteurs d"'Autoroutes de l'information" est d'abord formulé par leurs soins, et ensuite traduit dans la bouche des élus, qui reprennent à leur compte certains propos sans en prendre conscience. Ce système de relations entre les producteurs de savoirs et les personnels et élus des collectivités territoriales est voulu.

L'élu, censé être à l'aise sur ces questions, apprend souvent beaucoup, sans l'avouer parfois, car le savoir dans ce domaine est inégalement partagé, peu de choses sont réellement maîtrisées. Les élus s'intéressent à ces supports dans la mesure où l'information devient pour eux un savoir, sans même qu'ils s'en aperçoivent; ils recyclent ensuite ces données, mais toujours de manière non pensée, dans leur communication personnelle envers leurs personnels ou leurs 
concitoyens. Ce que les élus apprennent par ces supports, ils vont le traduire ailleurs.

Ce phénomène d'acquisition de savoirs informels rejoint le concept d"'opinion vraie", proposé dans La République de Platon, qui se différencie de celui de "connaissance". Elle ne peut rendre compte de sa vérité par raisonnement ou démonstration, elle est une connaissance pratique, donc, comme il y en a dans toute technique ou dans tout art, mais on ne sait pas vraiment où elle a pris sa source. Dans le domaine de l'art, celui qui reconnaît l'existence de la beauté absolue et qui est capable d'apercevoir à la fois cette beauté et les choses qui y participent, c'est l'homme qui connaît, c'est de la connaissance, c'est de la science. Celui qui jugera la beauté sur l'apparence, c'est de l'opinion. Il ne saisira que les apparences. Comment avoir les moyens de connaître quelque chose qui n'est pas ? Certaines choses sont, et ne sont pas. La connaissance se rapporte à l'être, l'ignorance au non-être.

Il existe un milieu entre l'ignorance et la science, c'est l'opinion, faculté distincte de la science, une force grâce à laquelle on fait ce que nous pouvons faire (vue, ouïe), mais on ne sait le décrire, et l'on ne regarde que son objet, ou ses effets. Elle est plus obscure que la connaissance, et plus lumineuse que l'ignorance, elle est intermédiaire. On a sur toutes choses des opinions, mais on n'a aucune connaissance de nos opinions. On dira qu' 'ils n'ont sur toute chose que des opinions, mais que des objets de leurs opinions, ils n'ont aucune connaissance"1. Autrement dit, nous serions devant une voie nouvelle d'apprentissage, qui est informelle. Cette opinion vraie se distingue de "l'opinion fausse", de l'apparence, de l'illusion, et de la science.

Kant, dans Critique de la faculté de juger, parle lui de l'art comme savoir-faire et habileté non théorisable, qui suppose une sorte de flair. L'art comme habileté de l'homme, est aussi distinct de la science (comme pouvoir est distinct de savoir). La faculté pratique est distincte de la faculté théorique. La technique est distincte de la théorie ${ }^{2}$.

1 Platon, La République, Paris, PUF, 1990, p. 244.

2 E. KANT, Critique de la faculté de juger, Paris, PUF, § 43, al. 2. 


\section{Médiatisation camouflée de savoirs}

Socialement, ce support a vocation à livrer de l'information, mais le découpage des rubriques laisse une grande place aux savoirs, dispensés sous forme de "dossiers", d"'abécédaires", etc. C'est donc bien aussi le mode de circulation des informations qui est informel, se définissant en marge des normes et modèles imposés par les systèmes habituels, proches d'une information plus factuelle.

\section{Banaliser, communiquer sur l'outil pour le renforcer}

La banalisation de l'outil technologique comme un savoir-faire abordable par tous, est un moyen choisi par les rédacteurs de la lettre pour donner aux interlocuteurs la sensation qu'il est facilement compréhensible, et qu'ils vont apprendre à l'utiliser sans même s'en rendre compte. Le but est de dédramatiser l'outil afin de calmer les réticences traditionnelles aux technologies: "longtemps considérés comme peu fiables, et compliqués, à destination d'un cercle d'initiés, les logiciels sont retenus par quelques collectivités territoriales comme des solutions" (AAI, $\mathrm{n}^{\circ} 44,15 / 02 / 99$ ). Comme si le propos était de montrer que les acteurs concernés étaient déjà dans cette mutation technologique, en train d'évoluer, sans s'en rendre compte.

La part communicationnelle compte pour que le dispositif pédagogique fonctionne. La réussite du dispositif du savoir est conditionnée par les interfaces assurant l'échange des messages entre apprenants et machines à enseigner. La communication a donc une fonction pédagogique à part entière. La pédagogisation des systèmes techniques est censée être venue par l'évolution de la société entière. La pédagogie devient fonction sociale par excellence.

\section{Enjeu de ces nouveaux dispositifs de savoirs : une médiation à la fois communautaire et interactionniste}

Sur les bases de cet examen de la forme et de la diffusion des contenus pédagogiques de cette publication, tentons d'examiner quelles recompositions ces savoirs spécifiques entraînent dans les relations entre les acteurs qu'ils touchent.

$\mathrm{La}$ modification des apprentissages a des conséquences anthropologiques, puisqu'elle contribue, en plus de l'instruction des élus, à une logique de formation des identités professionnelles, et à la 
structuration d'une sphère professionnelle. Les motivations profondes des concepteurs de la Lettre, par les mots qu'ils emploient, ne sont jamais neutres et contiennent souvent des stratégies porteuses d'une dynamique de production de sens.

Dans un premier temps, cette partie examinera les effets produits par cette mutation dans les savoirs : évolution des procédures de travail des élus et personnels territoriaux, apparition d'une médiation communautaire.

Dans un deuxième temps, nous examinerons en quoi leur place est redéfinie à l'intérieur de notre société, par l'ouverture du monde des collectivités territoriales à celui de l'expertise qui leur offre également une médiation interactionniste.

\section{Une médiation communautaire}

"Autoroutes de l'information et territoires" médiatise l'internet de manière orientée et influence les élus, dont les pratiques sociales préexistantes évoluent. Il n'existait pas de besoin dans ce milieu politique, mais l'offre a eu un rôle moteur, portée par un volontarisme industriel fort. Cette cristallisation des usages des nouvelles technologies a produit une norme sociale en même temps qu'elle a produit ces usages.

La cible de la lettre illustre clairement la volonté de regrouper une population autour d'un même discours' ${ }^{1}$. Elle touche les techniciens, non techniciens, fonctionnaires des collectivités territoriales, dans les directions informatiques, les services d'information des collectivités territoriales, les webmestres, les services d'information et système d'information, les cabinets d'élus politiques, les décideurs politiques, les chefs de services de communication.

Toute connaissance passe par des médiations dont le pouvoir de représentation est assez fort pour produire une certaine forme de réel ${ }^{2}$, grâce au discours, dont les termes fédérateurs sont tournés vers l'idée selon laquelle il faut installer un intranet dans toutes les collectivités, sous peine d'être dépassé.

R. Chartier écrit : "Il n'est pas de pratique, ni de structure qui ne soit produite par les représentations, contradictoires et affrontées, par

1 Ibid.

2 V. DE BRIANT et Y. PALAU, La médiation, définition, pratiques et perspectives, Paris, Éd. Nathan Université, 2001, p. 5. 
lesquelles les individus et les groupes donnent sens au monde qui est le leur"1.

L'usage de ce média n'est donc ni artificiel ni superflu. Il établit un lien entre les élus, en leur offrant le moyen de se connaître, venant en complément des salons, des formations qui remplissent aussi cette fonction fédérative, de constitution d'une identité professionnelle commune.

Par des éléments discursifs et symboliques, ce média encourage une certaine perception de la réalité des collectivités territoriales, unifiée, harmonisée, et finit par créer une norme propre à un monde social à l'intérieur duquel tous le lisent, en tirent des enseignements, des lignes de conduite, des orientations dans les choix à opérer en matière de nouvelles technologies.

Une médiation communautaire s'installe à partir de connaissances partagées autour d'un support donné, et sert d'instrument d'autorégulation pour une communauté, en essayant de mettre à sa disposition, en les harmonisant, des savoirs sur les technologies. Il favorise des relations qui n'étaient pas forcément existantes avant que ce média ne soit proposé, en véhiculant une certaine représentation d'internet et de la société, une nouvelle identité. Il répond à la crise des médiations sociales et traditionnelles.

Une telle médiation privée, de proximité, correspond à la mise en relation de plusieurs êtres qui nouent un lien social sur une base qu'ils définissent eux-mêmes afin de réguler une communauté, à l'intérieur de la sphère des collectivités territoriales.

Le vocabulaire relevé autour des savoirs technologiques passe du registre de l'explication à celui de la quasi-propagande. Avec parfois une tonalité culpabilisatrice, d'injonction à se former, précisant qu'il fallait "en être", rien n'étant plus normal.

La plupart des élus rencontrés au cours de nos entretiens dans ce secteur des collectivités locales sont unanimes : sans formation les acteurs échouent. "L'appropriation des technologies par l'ensemble des acteurs du secteur touristique des collectivités territoriales devient un facteur de compétitivité essentiel sans lequel le maintien des parts de marché de la destination France seront plus difficiles"2, et "aucun

1 R. Chartier, "Le monde comme représentation", Annales ESC, n 6, Paris, nov. 1989 , p. 1505.

2 Jean-Luc Fichet, conseiller général, élu du Finistère, Communauté d'Agglomération de Morlaix, entretien du 12 nov. 2000. 
emploi administratif n'échappera à la connexion au réseau" (AAI, $n^{\circ} 43,30 / 01 / 99$ ). La meilleure façon d'appréhender les changements autour des technologies de l'information et de la communication est d'en être un acteur à part entière.

Le "consensus" rencontré dans les décisions à prendre en matière de nouvelles technologies, "adoptées à l'unanimité, tous courants politiques confondus" (AAI, n 43, 30/01/99), est flagrant. De même, la naturalisation de l'outil s'impose: "les réseaux de villes ont naturellement vocation à développer les nouvelles technologies" (AAI, $n^{\circ} 43,30 / 01 / 99$ ), ou encore : "Bourges utilise Internet pour gérer plus efficacement l'ensemble des services municipaux" (AAI, $n^{\circ} 48,30 / 04 / 99$ ).

Il y aurait aussi un certain prestige à développer l'outil informatique dans "la ville de demain": intégrer les technologies dans l'aménagement de la ville semble indispensable, et de citer l'exemple de André Santini, qui évoque la possible "villa Médicis numérique", à la gloire de la domotique, pour Issy-les-Moulineaux. Métaphore qui vise à sacraliser les technologies (AAI, $\mathrm{n}^{\circ} 48,30 / 04 / 99$ ).

Le Livre vert sur la meilleure façon d'utiliser l'information, présenté par Martin Bangemann, membre Commission européenne sur question de société de l'information (AAI, $n^{\circ} 43,30 / 01 / 99$ ) contribue à cette vocation de publiciser les débats, de renforcer ce culte de l'internet, pour mieux avancer ensemble, montrer que c'est la solution idéale pour l'administration, que l'informatisation généralisée est vitale, et qu'elle permettra de mieux travailler -amélioration des conditions de travail par la technologie. "Nous sommes entrés dans l'ère de l'immatériel qui crée richesse et emploi, le web est devenu indispensable" (AAI, $\mathrm{n}^{\circ} 44$ 15/02/99).

Chacun se doit de relayer une idéologie de la circulation et de la transparence, de la symbolique, des signes et de l'imaginaire liés aux objets techniques eux-mêmes. De même qu'hier la société de consommation recherchait des signes de différentiation dans des objets, la société de communication joue aujourd'hui de la même partition mais avec les objets de l'information qui sont autant de signes de pouvoir ou de convivialité. Ainsi, le branchement sur Internet vaut-il une adhésion à une communauté virtuelle. 


\section{Une médiation interactionniste}

La logique corporative proposée par la Lettre se double d'une redistribution des pouvoirs. Les professionnels intervenant dans ce processus imposent leur point de vue face aux usagers et aux politiques.

Sans toutefois tomber dans le manichéisme qui renvoie dos à dos espaces public et privé, observons malgré tout que la vision de cette lettre d'information est bien celle d'acteurs privés. En s'exprimant, ils diffusent un avis particulier, offrent une représentation des acteurs des politiques locales qui sera celle partagée ensuite par le sens commun. Le multimédia est présenté comme "la solution pour revitaliser les écoles et les collèges". Les "sites portails valorisent un territoire et son économie" (AAI, n 42, 15/01/99).

Ces nouveaux acteurs donnent leur lecture de la situation, publicisent, médiatisent, donnent leur avis, livrent un diagnostic sans trancher, tout ceci participant à un discours dont les effets se feront sentir dans la population entière.

"Aujourd'hui, la capacité d'expertise et de décision publique des cadres communaux dépasse celle des maires", nous explique J-L. Fichet ${ }^{1}$, élu et convaincu de la réalité de ces changements. Dans la manière dont les décisions politiques sont implémentées, les choix vont vers la pluridisciplinarité. L'environnement des prises de décision est évolutif, et l'on entre dans un processus de contestation de primauté de l'élu, qui vise à affirmer une volonté d'anticiper le changement pour se légitimiter, pour définir une éthique, des valeurs, une identité.

Les objectifs des institutionnels s'orientent ainsi vers une approche pluridisciplinaire, pour la réflexion sur les politiques de la ville ou d'autres échelons, dans le vœu de favoriser une proximité entre le politique et les habitants, et de favoriser l'émergence d'une connaissance dédiée aux technologies, au progrès, au changement, à l'ouverture sur les environnements extérieurs grâce à l'analyse prospective. Le but est d'enseigner, certes, mais aussi de convaincre les élus et les habitants du bien fondé des technologies.

1 Ibid. 


\section{Conclusion}

"Autoroutes de l'information et territoires" veut transmettre des connaissances, rendre compte des usages technologiques dans les collectivités locales, afin de banaliser et de donner un caractère reproductible aux pratiques qui ont cours sur un territoire donné.

L'information sur les technologies de pointe se diffuse donc imperceptiblement sur ce support spécifique. L'enseignement est alors associé et réduit à l'outil même de communication, ce qui crée une coproduction de sens. Au-delà du simple élargissement de la fonction de formation, l'utopie de la société pédagogique est bel et bien censée offrir les conditions et les moyens de leur ressaisissement ${ }^{1}$ à un espace public et un fonctionnement sociétal privés de leurs anciennes légitimités.

L'accès à la technologie reste pourtant discriminatoire, sur un plan technique comme financier ou géographique. Cette situation remet alors en cause l'une des données d'origine de l'information municipale, celle qui a contribué à asseoir sa légitimité : le droit pour chaque habitant à l'information sur les décisions municipales.

Or, la pression pour l'innovation et les mutations techniques, très forte ces dernières années, n'oblige-t-elle pas les collectivités locales à entrer dans une situation de monopole de l'information et de la communication? Elles sont en effet tout entières absorbées par le devoir de se plier à l'apprentissage des techniques plutôt que par celui de consacrer du temps aux citoyens.

Il ne faut pourtant pas céder à la tentation des généralités sur la recomposition de l'espace public, et ne pas avoir non plus de vision substantialiste de la technique. Admettons plutôt l'idée de la non dégradation de l'espace public. Il s'avère simplement occupé par de nouveaux outils cognitifs, mais dont la liberté de manœuvre n'est pas aussi importante que ce qui a pu être écrit ou dit parfois.

1 P. MoEGLin, Congrès SFSIC, Lille, 1996, p. 24. 